UCRL-JC-121626

PREPRINT
CONF-9508168--1

\title{
High-Power Copper Vapour Lasers and Applications
}

\author{
J. J. Chang \\ B. E. Warner \\ C. D. Boley \\ E. P. Dragon
}

This paper was prepared for submittal to Advanced Research Workshop

St. Andrews, Scotland, UK

August 7-11, 1995

August 1995

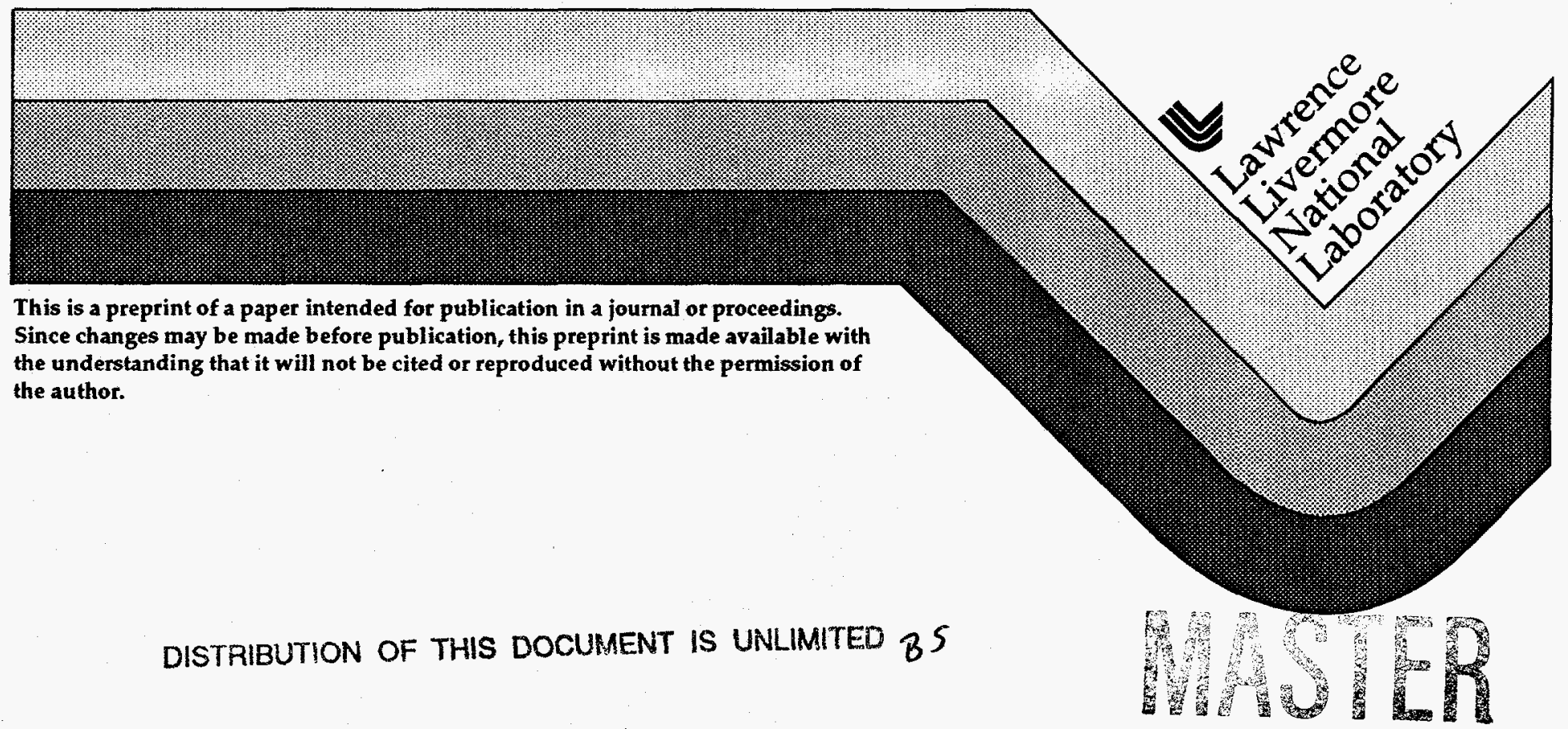




\section{DISCLAIMER}

This document was prepared as an account of work sponsored by an agency of the United States Government. Neither the United States Government nor the University of California nor any of their employees, makes any warranty, express or implied, or assumes any legal liability or responsibility for the accuracy, completeness, or usefulness of any information, apparatus, product, or process disclosed, or represents that its use would not infringe privately owned rights. Reference herein to any specific commercial product, process, or service by trade name, trademark, manufacturer, or otherwise, does not necessarily constitute or imply its endorsement, recommendation, or favoring by the United States Government or the University of California. The views and opinions of authors expressed herein do not necessarily state or reflect those of the United States Government or the University of California, and shall not be used for advertising or product endorsement purposes. 


\section{DISCLAIMER}

Portions of this document may be illegible in electronic image products. Images are produced from the best available original document. 


\title{
HIGH-POWER COPPER VAPOUR LASERS AND APPLICATIONS
}

\author{
J. J. Chang, B. E. Wamer, C. D. Boley, and E. P. Dragon \\ Lawrence Livermore National Laboratory \\ P.O. Box 5508, M/S L-463, Livermore, CA, 94551, USA
}

\begin{abstract}
Expanded applications of copper vapor lasers has prompted increased demand for higher power and better beam quality. This paper reports recent progress in laser power scaling, MOPA operation, beam quality improvement, and applications in precision laser machining. Issues such as gas heating, radial delay, discharge instability, and window heating will also be discussed.
\end{abstract}

\section{Introduction}

The applications of high-radiance, short-pulse copper vapor lasers (CVL) in second harmonic generation [1,2], precision laser machining [3.4], and laser beacon [5] have prompted increased demand for diffraction-limited high-power CVL. The CVL lasers developed mostly for atomic vapor laser isotope separation (AVIS) are not quite suitable for these applications mainly because of their large beam divergence. Furthermore, the need of a simple multi-hundred-watts master-oscillator-poweramplifier (MOPA) systern for most industrial applications makes the power scaling of a CVL amplifier more important. In the past years, LLNL has developed several new technologies that have successfully expanded high power CVL's to non-AVLIS applications. This paper reports our works on CVL power scaling and beam quality improvement. High-power CVL applications in precision laser machining and second harmonic generation will also be discussed.

\section{Near-diffraction-limited Oscillator}

A near-diffraction-limited CVL oscillator is essential to achieve a low-divergence highpower CVL output through amplification. A conventional CVL unstable resonator is not suitable because only the latter part of the output pulse reaches near-diffractionlimited beam quality $[6,7]$. The use of a self-filtering unstable resonator [8], although has generally improved CVL resonator beam quality, its output pulse still leads by a large amount of ASE that shortens it pulse and lowers its usable low-divergence laser light for efficient amplifier extraction. To circumvent these difficulties, injection locking techniques [9.10] has been applied to obtain low divergence output for the entire duration of the laser pulse. 
Figure 1 illustrates an injection controlled oscillator used to achieved neardiffraction-limited output for high-power amplifier extraction. A 5-10 w low-divergence injection beam is generated with a 2-cm bore master oscillator (MO) that has a confocal negative-branch unstable resonator with magnification 100 . It's beam quality is 2 times diffraction limited (XDL) based on a power-in-the-bucket measurement [7], which is similar to a $\mathrm{M}^{2}$ knife-edge method. Note that a self-filtering unstable resonator is not appropriate for MO because of the possibility injection feedback that may generate parasitic oscillation and damage the intracavity pinhole. The $4-\mathrm{cm}$ bore injection controlled oscillator (ICO) is a self-imaging unstable resonator with magnification 15 . The cavity relays the feedback aperture to the laser output on the scraper mirror that effectively reduces the diffraction effect of the hard aperture and substantially improves ICO output beam quality. A time delay is applied between the discharges of $M O$ and the ICO such that the ICO gain is only seeded by the latter part of the MO ouput that has a better beam quality; the ICO is essentially a free-running oscillator after the injection beam termipates itself.

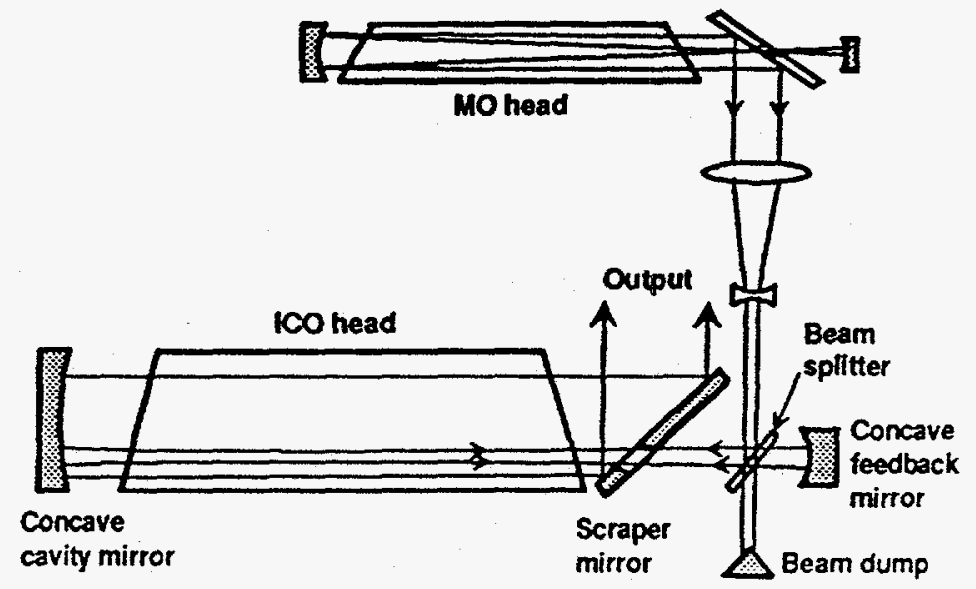

Fig. 1. A schematic layout of the injection controlled oscillator.

The demagnified MO beam is injected into the ICO cavity via a beam splitter inside the ICO cavity, as shown in Fig. 1. An adjoint-coupled injection scheme is applied to matches the injection signal with the ICO adjoint mode (i.e., convergent mode). In this scheme the collimated injection beam, upon reflecting from the concave cavity mirror, first travels inward but then gradually spreads out because of Fresnel diffraction. This process speeds up cavity mode formation and the conversion of the injection signal to an ICO cavity mode becomes possible before the ICO laser gain starts. To avoid strong pre-pulse plasma absorption and to take the advantage of CVL radial delay, an off-axis unstable resonator is applied to accommodate an off-axis injection as illustrated in Fig. 1. In this scheme, the seeding beam is injected into the cavity along the wall of the plasma tube where the laser gain starts earlier and the plasma absorption is weaker. To obtain a better seeding signal, the $2-\mathrm{XDL}$ injection beam is improved through a spatial 
filtering technique that accommodates the injection and ICO cavity configurations. In this method the large concave cavity mirror and the small feedback aperture on the scraper serve as a spatial filtering system. The size of the injection beam is controlled such that its diffraction-limited spot size, focused by the large concave mirror, is the same as the hole size on the scraper, which is a spatial filter and a cavity feedback aperture. This technique effectively improved the seeding signal that lead to an 100 beam quality better than that of MO. Figure 2 illustrates pictures of two reverseshearing fringes of the ICO output with and without injection control. The improvement of beam spatial coherence with injection is evident and the fringe visibility of Fig. $2 \mathrm{~b}$ is nearly 1 even for the largest shear. A power-in-the-bucket measurement indicated that $\sim 84 \%$ of $1 C O$ output is within $1.3 \mathrm{XDL}$ beam divergence.

Without injection

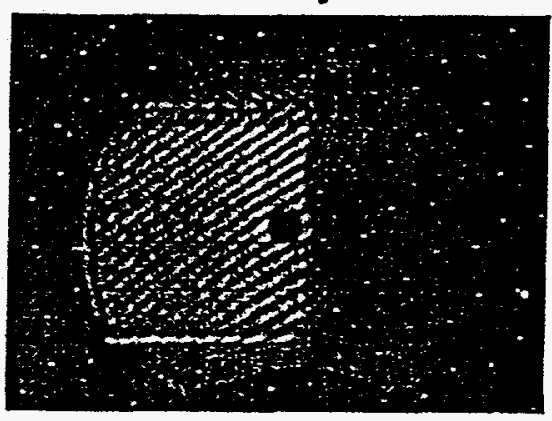

With injection

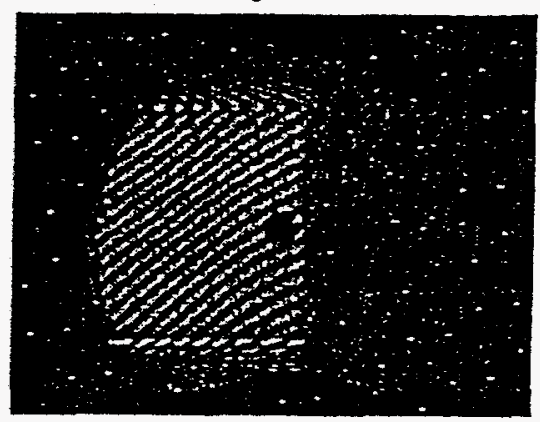

Fig. 2. Shear fringes of the 4-am ICO ouput beam measured with lateral reverse shearing techniques, (a) without injection, (b) with injection. Due to limited size of shearing optics, only half of the beam were sheared.

\section{High-Power Amplifier}

Expanded applications of high-power CVL has prompted considerable effort directed towards improving their performance. Volumetric scaling for higher laser power can be attempted either by lengthening the discharge tube or by increasing the wbe diameter. Length scaling, however, is limited by high voltage constraints and pulsed power technology. Attempts to scale in diameter are limited by excessive central gas heating, which degrades the laser efficiency by thermally populating the metastable lower laser levels, $2 D_{3 / 2}$ and $2 D_{5 / 2}$ states [11]. In addition, the decreased central gas density lowers the plasma impedance, thus lengthening the time for the discharge field to diffuse to the center. This leads to insufficient axial pumping and a non uniform beam profile. The increase input power requirement also put significant thermal load on discharge electrodes that worsens discharge instability, resulting large discharge jitters, severe window contamination, and short electrode lifetime. In addition, the large 
window heating resulted from either window contamination or tube IR radiation introduces severe amplifier wavefront distortion that limits their applications. Recently, significantly progress has been developed to overcome these issues. .

\subsection{GAS TEMPERATURE AND RADIATIVE COOLING}

A new method have been developed recently to reduce gas heating of large-bore CVL and to improve their performance through radiative cooling [12]. In this approach, a number of segmented plates (septa) are placed along the length of the tube, as illustrated in Fig. 4. The efficient radiative heat exchange between the side wall and the septa effectively lowers the septa temperature close to the tube temperature. The gas in the axial region is then in turn cooled through heat conduction to the septum plates.

In a CVL, the gas temperature changes litule during a pulse because of the large thermal inertia of the gas. The gas temperature distribution $T(r)$ can thus be described by the steady state heat conduction equation,

$$
\nabla \cdot(k \nabla T)=-P,
$$

with $k$ the conductivity of buffer gas, $P$ the time-averaged power per unit volume deposited the gas, given by a sum over momentum transfer collisions between electrons and heavy species. Under the reasonable assumptions of uniform power deposition and uniform wall temperature, one can calculate the temperature profile. The difference between a $C$ laser and a $S$ laser is illustrated in Figure 1, which shows calculated radial temperature distributions at 75 tor buffer gas pressure (Ne with $0.5 \% \mathrm{H}_{2}$ ). The presence of septa lowers the central temperature by about $1370 \mathrm{~K}$, and it lowers the temperature at $a / 2$ (above the radial center of a septum) by $440 \mathrm{~K}$. In the case without septa, the profile calculations agree with the measured copper ground state density

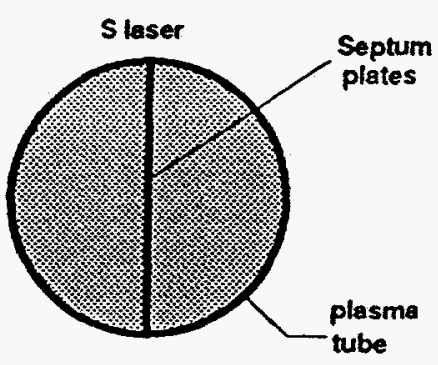

Fig. 4. Schematies of the cross secions of a sepum laser ( $\mathrm{S}$ laser).

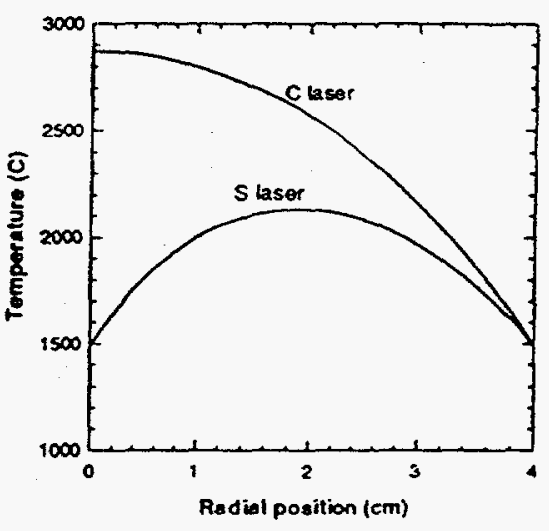

Fig. 5. Computed gas temperature profite of a $C$ laser and a $S$ laser at a buffer gas pressure of 75 tor 
profile [2]. The decreased gas temperature associated with the septa plays two beneficial roles. First, the prepulse electron temperature is allowed to equilibrate to a bwer value, thus lowering the prepulse metastable densities. Second, the increased gas density gives rise to an increased discharge impedance. This is manifested, for example, in a storter radial delay in field penetration and a better coupling between the laser head and the pulse power modulator.

\subsection{FLELD PENETRATION}

Because of the high conductivity nature of CVL plasma, the plasma skin effect predominates the initial stage of its gas discharge. The effect of gas temperature and gas pressure on field penetration follows from the behavior of the resistivity, as manifested in the field diffusion equations. In the absence of axial gradients, the field components are $B_{\theta}(r, t)$ and $E_{z}(r, t)$. The magnetic field diffuses according to

$$
\frac{\partial B_{\theta}}{\partial t}=\frac{\partial E_{z}}{\partial r}, \quad E_{z}=\frac{D}{r} \frac{\partial\left(r B_{\theta}\right)}{\partial r}
$$

where the diffusion coefficient $D=\rho / \mu_{0}$. The penetration time varies inversely with the resistivity, $\rho=m_{e} v / e^{2} n_{e}$, which in turn is proportional to the electron momentum transfer frequency $v$ and inversely proportional to the electron density $n_{e}$. During the pulse, $v$ is dominated by electron-neon collisions and hence increases as the gas density is increased at lower temperature or higher pressure. In addition, our simulations show that, during the time of initial field penetration, the electron density is closer to its quiescent value than to its peak value, and that the latter is not too sensitive to the gas temperature. Since a lower gas temperature gives rise to a lower quiescent electron density, this is another mechanism for raising the resistivily. According to the model, the two effects are of comparable importance. The effect of a cooler plasma and higher gas pressure on field diffusion is illustrated in Fig. 6, which shows the measured redial delay for both $C$ laser and $S$ laser. The reduction in radial delay at higher pressures and lower gas temperatures (i.e., $S$ laser) shown is mainly a result of higher gas density [13].

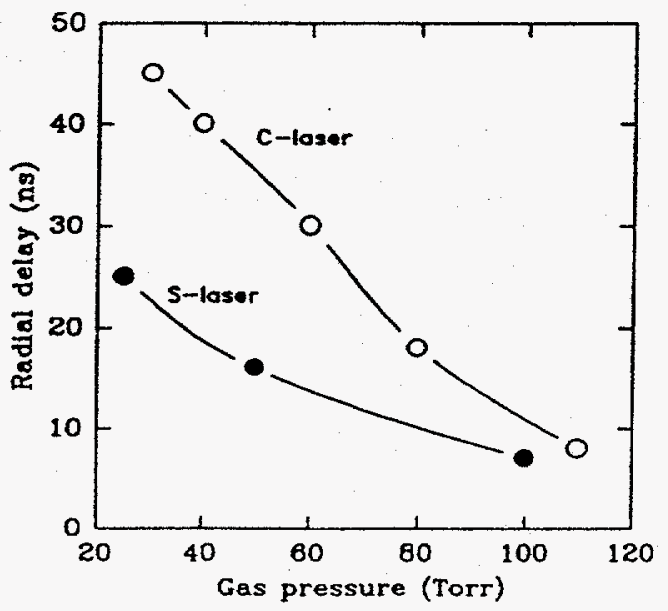

Fig. 6. Comparison of measured redial delay between an $S$-laser and a $C$-laser at various buffer gas pressures. 


\subsection{SATURATION FLUENCE AND LASER POWER}

This amplifier was driven by a $4.4 \mathrm{kHz}$ pulse modulator with 3-stage magnetic compression that delivered a peak voltage of approximately $40 \mathrm{kV}$ with a rise time of less than 50 ns. The determine the S-laser saturation flurence, its energy has been extracted with various signal intensity as illustrated in Fig. 7. It shows that the extracted energy levels of at injection of approximately $4 \mathrm{~mJ}$ for the yellow output (578 $\mathrm{nm})$ and $6 \mathrm{~mJ}$ for the green output $(511 \mathrm{~nm})$. It translates to injection fluences of $-80 \mu$ $\mathrm{J} / \mathrm{cm}^{2}$ for the yellow and $120 \mu \mathrm{J} / \mathrm{cm}^{2}$ for the green are required for efficient energy extraction of the 8-cm amplifier.

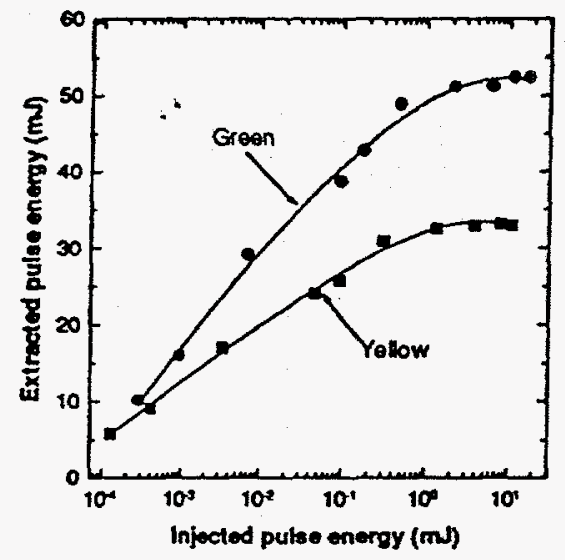

Fig. 7. Measured energy extraction at various injection energy for both green $(511 \mathrm{~nm})$ and yellow $(578 \mathrm{~nm}$ ) output. The solid lines are least-square curve fiting of the data points.

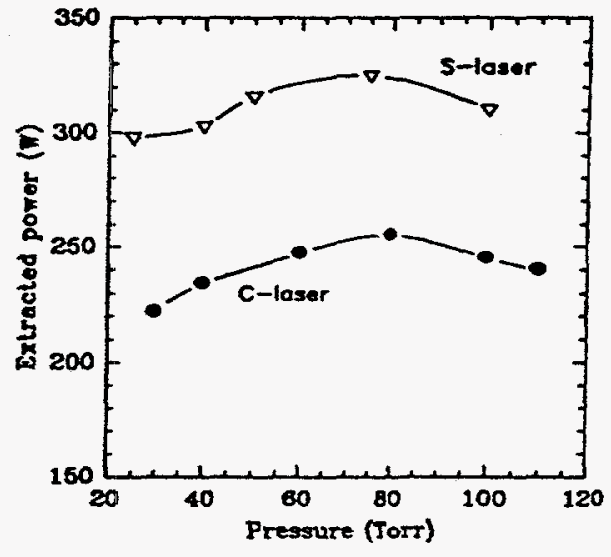

Fig. 8. Extracted output power of 8-an copper laser amplifiers with septa (S-laser) and withour septa (C-laser) at different buffer gas pressures.

Figure 8 illustrates the measured amplifier power of both $S$ and $C$ lasers. In this experiment, the optimized laser input power needs to be lowered at higher pressures because of increased head impedance. The optimized gas pressure for both cases are between 70-80 torr. At this optimized lasing condition, the extracted laser power increased from $255 \mathrm{~W}$ with a C-laser to $325 \mathrm{~W}$ with an S-laser, a $27 \%$ improvement. A small reduction $(-5 \%)$ in required input power was also observed when septa were used. This is expected because of lower gas temperature associated with the $S$ laser that improved coupling between the laser head and the pulse modulator. An improvement of laser efficiency from $1.1 \%$ to $1.38 \%$ was achieved with the addition of septa, based on the energy stored in the front capacitor. As a final remark, Iscki et. al. recently reports a nearly $57 \%$ increase of laser power (i.e., running with a plan-plan resonator) with an 8$\mathrm{cm} \mathrm{CVL}$ by the addition of two cooling plates [14]. Their measurement of $\mathrm{Cu}$ ground state density confirms the reduction of axial gas temperature with the addition of septa. 


\subsection{ELECTRODES AND WINDOW CONTAMINATION}

The peak discharge current of a CVL is typically very high for efficient pumping. For example, it's about $2.5 \mathrm{kA}$ for the 8 -cm 300 -W amplifier. The large discharge current induces an abnormal cathode fall as high as 1-2 kV within a cathode sheath that is smaller than $100 \mathrm{\mu m}$ [15]. The inevitable high discharge impedance using conventional electrodes leads to a large amount of thermal energy deposited into the cathode-fall region within a time duration of 200-500 ns. Additionally, the advancement of pulse-power electronics enables CVL amplifiers to be operated at higher Ne pressures that put more stress on electrodes because the volumetric thermal loading at cathode increases with gas pressure. This high rate of energy deposition is prone to thermal instability [16] that usually results in constricted discharge at the cathode accompanied with severe electrode sputtering and erosion. This phenomena in a CVL degrades the laser beam quality due to induced window contamination and instability of far-field spot. The discharge instability also leads to large discharge jitters (10-20 ns) that lowers amplifier extraction and causes fluctuation of MOPA output energy..

One approach to resolve these problems is to applied hollow cathode discharge because of its more efficient generation of free electrons for a high-current discharge. However, conventional hollow-cathode electrodes were mostly applied to low-power, low-pressure devices because of the requirement of small hollow cathode space that limits the electrode size. A new type of hollow cathode was thus developed [17] for high-power CVLs that typically operated at 40-100 torr of buffer gas pressures. The electrode employs a uniform-field profile that significantly enlarges the effective area for discharge. Although it's generally believed that a profiled electrode is not necessary for longitudinal discharge devices, the short-pulse high-peak-current discharge of a CVL distinguishes itself from conventional longitudinal discharge that have either $\mathrm{CW}$ or long-pulse operation. We found the electrode profile is essential to improve the CVL discharge stability and electrode lifetime. Multiple hollow-cathode slots were applied to the profiled electrode surface, as illustrated in Fig. 9, to further improve electron emission. Copper was used for the electrode construction because of its lower fabrication cost and less oxidization induced window contamination than refractive materials.

This new electrode design not only maintains the need of small hollow cathode space for efficient hollow-cathode discharge at elevated gas pressure, but also satisfied the high-power requirement by applying multiple slots. The gap size of slots is designed to have effective hollow-cathode effect under CVL operational gas pressure but with minimized discharge erosion. A gap size of a fraction of $\mathrm{mm}$ was found to be adequate for effective electron emission and electrode lifetime. Our experiments indicates that discharge constriction on a profiled electrode without hollow-cathode slots started at about 30 torr. The addition of multiple slots effectively extended the stable glow discharge to -100 torr. Window contamination and discharge jitters were greatly reduced with this new electrode design. The lifetime of this multi-slot hollow cathode electrode has been found to be around 15,000 hours for 8 - $\mathrm{cm}$ amplifiers. 


\subsection{WINDOW HEATING AND AMPLIFIER BEAM QUALITY}

The installation of hollow-cathode copper electrode has eliminated most of the window contamination induced window heating by laser light. However, the unavoidable $\mathbb{R}$ radiation from the high-temperature plasma tube introduces noticeable window heating that results in beam focus and wavefront aberration, if care is not taken for the window design. Fused silica windows, due to their lower cost and negligible laser light absorption, has been widely used in CVLs. Nonetheless, the relatively high-IR absorption of fused silica makes it not suitable for applications that require neardiffraction-limited output. To diminish $\mathbb{R}$ heating, $\mathrm{CaF}_{2}$ and $\mathrm{MgF}_{2}$ windows are better suited than fused silica windows. IR transmission range (i.e., greater than 50\% transmission) for a 1 inch thick window are $8.8 \mu \mathrm{m}$ and $6.8 \mu \mathrm{m}$ for $\mathrm{CaF}_{2}$ and $\mathrm{MgF}_{2}$. respectively. It's considerably better than a fused silica window that also has an $-0.5 \mu \mathrm{m}$ $\mathrm{OH}$ absorption band centered at $2.7 \mu \mathrm{m}$. As listed in Table 1 , both $\mathrm{CaF}_{2}$ and $\mathrm{MgF}_{2}$ have low absorption in CVL wavelengths. They are almost insoluble in water and will remain an optical polish with no deterioration under normal atmospheric conditions. With a typical tube temperature of $1700 \mathrm{~K}$, it is estimated that $40 \%$ of the tube radiation that reaches the window is absorbed when a fused silica window is used (i.e., the $\mathrm{OH}$ absorption band absorbed $10 \%$ of the radiation). This absorption translates to a heat deposition of $4 \mathrm{~W}$ to the laser window, assuming an 8-cm plasma tube and a distance of $60 \mathrm{~cm}$ between the window and the tube opening. This heat deposition reduces to $0.6 \mathrm{~W}$ and $0.3 \mathrm{~W}$ for $\mathrm{MgF}_{2}$ and $\mathrm{CaF}_{2}$, respectively. The window heating on $\mathrm{MgF}_{2}$, although is slightly higher than $\mathrm{CaF}_{2}$, its high thermal conductivity and low $\mathrm{dn} / \mathrm{dT}$ represents significant advantages in lowering window heating induced beam focus and wavefront distortion. $\mathrm{MgF}_{2}$ is also more resistant to color center that may appear in $\mathrm{CaF}_{2}$ due to large amount of $\mathrm{UV}$ radiation from the laser plasma. Most $\mathrm{MgF}_{2}$ windows are cut perpendicular to its optical axis due to its birefringence characteristic. Sapphire windows, although has the highest thermal conductivity, is not considered because of its high $\mathrm{dn} / \mathrm{dT}$ and the cost of material and optical polish.

Table 1. Comparison of window materials for CVL [18]

\begin{tabular}{|c|c|c|c|c|c|c|c|}
\hline Material & $\begin{array}{c}\text { Thermal } \\
\text { conductivity } \\
(\mathrm{W} / \mathrm{m} / \mathrm{K})\end{array}$ & $\begin{array}{l}\text { Refracaive } \\
\text { index } \\
\text { a } 500 \mathrm{~mm}\end{array}$ & $\begin{array}{c}\text { Dn/DT } \\
\left(10^{-6 / K)}\right.\end{array}$ & $\begin{array}{l}\text { Cir-off } \\
\text { wavelength } \\
\text { a sox } T^{*} \\
(\mathrm{gm})\end{array}$ & $\begin{array}{l}\text { Esimaled } \\
\text { window } \\
\text { absompion ** } \\
\text { (W) }\end{array}$ & $\begin{array}{c}\text { Absorpion } \\
\text { coefficient at } \\
500-600 \mathrm{rm} \\
\left(10^{-5} \mathrm{~km}\right)\end{array}$ & $\begin{array}{l}\text { Radiation } \\
\text { resistance } \\
\text { (color } \\
\text { center) }\end{array}$ \\
\hline Fused silica & 1.38 & 1.462 & 12.8 & 3.3 & $4(40 \%)$ & 17 & Very good \\
\hline $\mathrm{CaF}_{2}$ & 9.71 & 1.437 & -10.6 & 8.8 & $0.3(39)$ & 7.2 & Fair \\
\hline $\mathrm{MgF}_{2}$ & 21 & $\begin{array}{l}1.383(\mathrm{O}) \\
1.395(\mathrm{E})\end{array}$ & $\begin{array}{l}2.1(\mathrm{O}) \\
1.7(\mathrm{E})\end{array}$ & 6.8 & $0.6(6 \%)$ & & Good \\
\hline Sapphire & 27.21 & 1.77 & 13 & 5.5 & $1(10 \%)$ & & Excellent \\
\hline
\end{tabular}

- Assuming a window thickness of 1 inch.

** Assuming a distance of $60 \mathrm{~cm}$ between the window and the $8 \mathrm{~cm}$ wbe opening

We have tested and compared fused silica, $\mathrm{MgF}_{2}$ and $\mathrm{CaF}_{2}$ windows in terms of window focusing and wavefront distortion by using wavefront shearing tochnique. The near-diffraction-limited oscillator described in Section 2 was used for the amplifier 
extraction. An window thermal isolator between the aluminum housing and the window was added to lower the window edge cooling and flatten its radial temperature profile. Table 2 lists the wavefront curvatures based on measured shear fringes of $x$-axis and $y$ axis of the output beam for fused silica, $\mathrm{CaF}_{2}$, and $\mathrm{MgF}_{2}$ windows.

Teble 2 Meatured window focus of the 8-con anplifier with different window materials

\begin{tabular}{|l|c|c|c|}
\hline Material & Fused Silic & CaF $_{2}$ & MpF \\
\hline X-axis wavefront radius $(m)$ & 250 & 850 & - \\
\hline Y-axis wavefront madius $(m)$ & 360 & 1200 & - \\
\hline P-V astigmation* & 1.6 wrves & 0.5 waves & 0 \\
\hline
\end{tabular}

- Extimated baced on difierem X \& Y wavefrom curvanres.

The window heating induced astigmatism, as shown in Table 2 , is believed mainly a result of free convection cooling of the $\mathbb{R}$ heated window (i.e., 10-15 K higher than the ambient air) that in effect shifts and tilts the curved wavefront. Table 2 shows that the improvement of output wavefront with $\mathrm{MgF}_{2}$ is significant because of its smaller window heating, larger thermal conductivity, and lower $\mathrm{dn} / \mathrm{dT}$. The higher $\mathrm{dn} / \mathrm{dT}$ of $\mathrm{CaF}_{2}$ windows made it not as effective as $\mathrm{MgF}_{2}$ windows for wavefront improvement. Based on shear fringe patterns, some window heating induced spherical aberration still exist even with $\mathrm{MgF}_{2}$ windows. A C-laser amplifier with $\mathrm{MgF}_{2}$ windows was extracted with a magnified ICO beam. Its far-field spot illustrated in Fig. 10 demonstrates a good beam focusability. The far-field energy spread measured with pinholes indicates larger than diffraction limited energy spread, as plotted in Fig. 11 at different laser power levels. Note that some wavefront distortion was introduced by the oscillator beam expander. At nearly full power level, $50 \%$ of the output has a beam divergence of diffraction-limited and about $84 \%$ output is within $2.2 \mathrm{XDL}$ beam divergence. This is a significant improvement over amplifier with fused silica windows, which had a beam quality of 5-6 XDL.

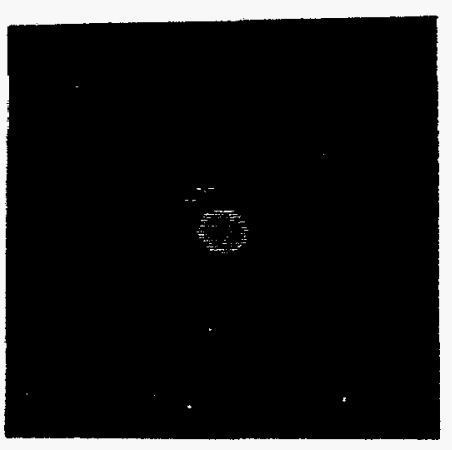

Fig. 10. A picuure of the amplifier far-field spol

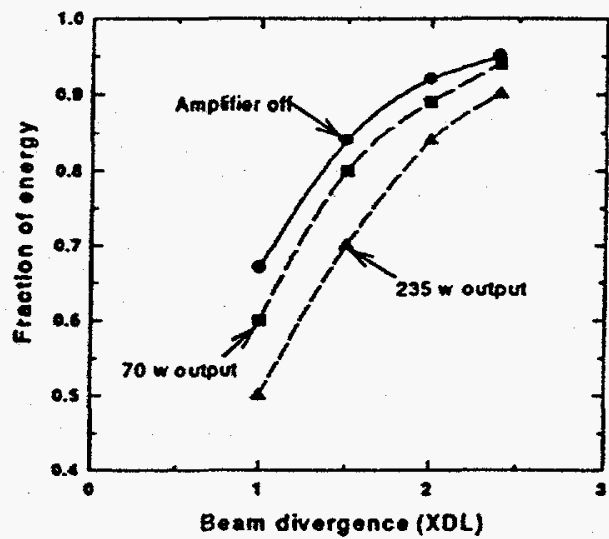

Fig. 11. Power-in-the-bucket measurement of the 8.cm amplifier far-ficld spor at various power levels 


\section{Applications}

\subsection{PRECISION LASER MACHINING}

Most industrial laser machining systems are based on $\mathrm{CW}$ or long pulse $\mathrm{CO}_{2}$ and YAG laser and material removal with these lasers is mostly through melt expulsion. This material removing mechanism normally leads to poor dimensional control and sizable recast layer that are not acceptable in precision machining applications. CVL provides a significant opportunity for precision laser machining $[3,4]$ because of its near-diffraction limited beam quality, short pulse output, and high-repetition frequency. A peak CVL power density of $10^{8} \mathrm{w} / \mathrm{cm}^{2}$ to $10^{10} \mathrm{w} / \mathrm{cm}^{2}$ can normally be obtained on material surface. This power density effectively removes material in a ablative fashion such that little recast layer is formed on the material surface. The short pulse also ensures micron scale heating depth and little heat affected zone has been observed. The CVL visible output greatly improves the laser-material coupling over $\mathbb{R}$ lasers because of higher surface absorption and lower plasma shielding.

Figure 12 illustrates CVL drilled $185-\mu \mathrm{m}$ holes on $1 \mathrm{~mm}$ thick steel based on a precision trepanning technology [3]. The holes are straight and their repeatability are excellent with hole size variation in the order of a few microns. Sections of CVL drilled holes show little recast layer or heat affected zone. Fig. 13 illustrates laser milled grooves on a piece of silicon carbide (i.e., $1.25 \mathrm{~mm}$ thick) with a high-power CVL

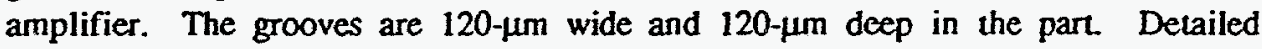
examination of the grooves showed straight side walls and fairly flat bottom. The holes in the part have diameters of 1.25 and $2.5 \mathrm{~mm}$. The hole roundness is nearly perfect and no material crack has been observed. Micromachining on alumina and silicon nitride has also been demonstrated with promising result.

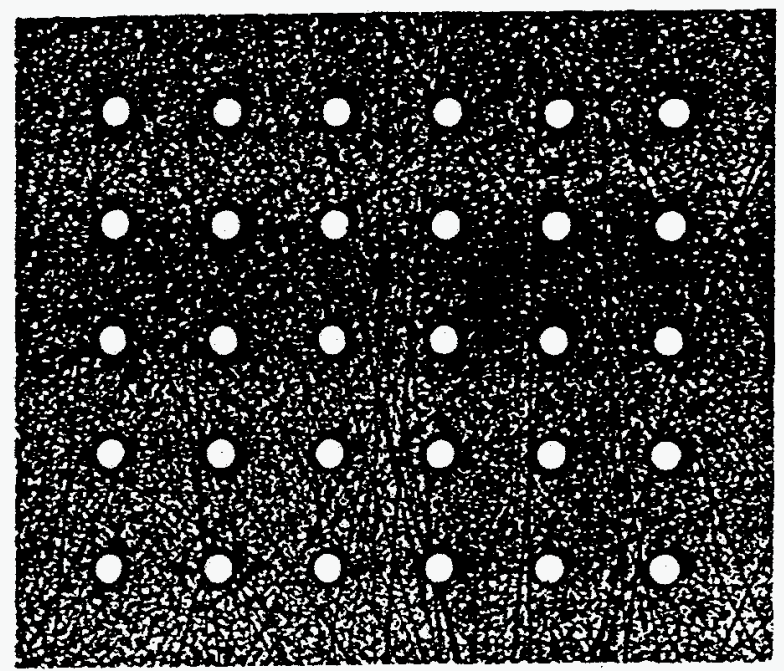

Fig. 12 Precision ucpanned holes with $185 \mu \mathrm{m}$ diameter on I-mm thick stecl using injection controlled CVL oscillator. 


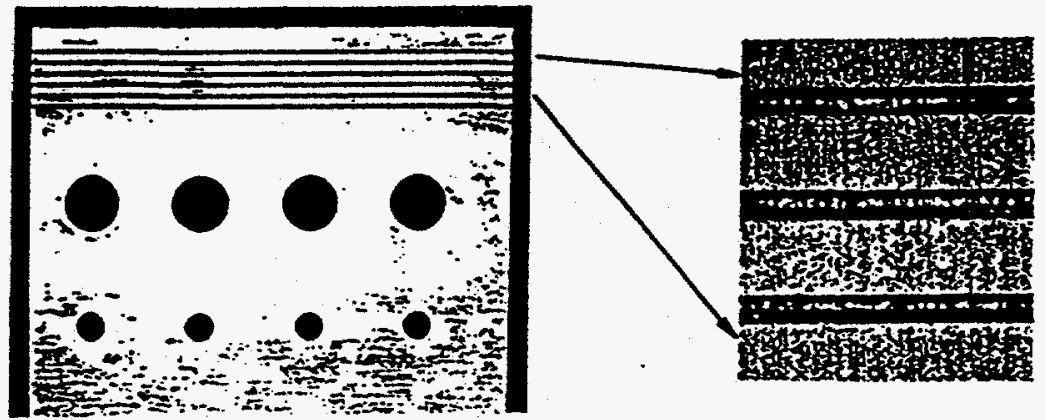

Fig. 13. Precision machined grooves and holes on a pioce of silicon carbide using a high-power CVL amplifier. The hole diameters are 1.25 and $25 \mathrm{~mm}$, the grooves are $120 \mathrm{\mu m}$ wide by 120 um deep.

\subsection{SECOND HARMONIC GENERATION}

The ability to generate high average power UV by frequency doubling the CVL output would greatly extend its applications in material processing [19] and microlithography. The frequency doubled $\mathrm{CVL}$ could replace $\mathrm{KrF}$ laser, which has a similar wavelength $(248 \mathrm{~nm})$ as doubled CVL $511 \mathrm{~nm}$. The doubled CVL's output has high-repetition frequency, near-diffraction-limited beam quality at a competitive cost. Second harmonic generation of an injection controlled CVL oscillator and a high-power amplifier has been demonstrated. A conversion efficiency of $18 \%$ has been achieved for the $511 \mathrm{~nm}$ output of ICO with a single BBO crystal [2]. This conversion efficiency was improved to nearly $30 \%$ with dual crystal oriented in altemating-z configuration [20]. Conversion efficiency of the $8-\mathrm{cm}$ amplifier (i.e., with fused silica windows) output drops to less than $10 \%$ because of beam quality degradation and severe thermal dephasing in the crystal. However, a record $9.0 \mathrm{w} 255 \mathrm{~nm}$ output has been demonstrated with $113 \mathrm{w}$ amplifier $511 \mathrm{~nm}$ output [20].

\section{Conclusion}

Significant progress has been achieved in the past years in CVL beam quality improvement, power scaling, and system reliability. These advancements have put high-power CVL in a very competitive position in entention to non-AVLIS applications such as laser material processing and UV generation. However, further system improvement is still needed to make CVL a widely acceptable laser tool. Issues such as maintenance cost (i.e., copper reload, tube lifetime), time requirement for laser on and off, and reliability in CVL pulse-power electronics are some of the main concerns normally raised by potential users. Improvement in these areas will greally enhance $\mathrm{CVL}$ in expanding its applications 


\section{Reference}

1. Cours, D., Ainsworth, M. and Piper, J. (1990) Enhancod efficiency of UV secood barmonic and aum frequency generation from copper vapor lasers, IEEE J. Quantum Electron. QE-26, 1555-1558.

2. Molander, W., Norton, M. and Chang, J. (1991) Improved efficiency of scoond-harmonic generation in BBO using the $511 \mathrm{~nm}$ line of an injection-seoded copper laser. Digest of Conference on Lasers and ElectroOptics, 176.

3. Chang, J., Martinez, M, Wamer, B., Dragon, E., Huete, G, and Solarski, M. (1995) Precision micro drilling with oopper vapor lesers. Proceeding ICALEO' $94,323-332$

4. Knowles, M. Kearsky, A. Foster-Tumer, R, Abbot, J., Baaler, J., and Errey, K (1994) Visualization of small hole drilling using a copper laser, Proceeding ICALEO'S4, 352-361.

5. Fugate, R. Fried, D. Amoer, G., Bocke, B. (1991) Measurement of atmospheric wavefront distortion using scaulered laser light from a laser guide-star, Nature 353, 144-146.

6. Omatsu, T. and Kuroda, K (1992) Time resolved measurement of spatial coherence of a copper vapor laser using a reversal shear interferometer, Opt. Commu. 87, 278-286.

7. Chang. J. (1994) Time-resolved beam quality characierization of copper-vapor lasers with unstable resonators, Applied Optics 33, 2255-2265.

8. Gobbi, P. and Reali, G. (1984) A novel unstable resonalor configuration with a self filtering eperture, Opt. commu. 52, 195-198.

9. Nayor, G., Lewis, R, and Kearsley, A. (1988) SPIE 894, 110.

10. Chang, J. (1995) Copper laser oscillator with adjoint-coupled self-filtering injection, Optics Letter 20, 575 577.

11 Wamer, B. and Kushner, M. (1983) Langebore copper-vapor lasers: kinetics and scaling issues, J. Appl. Phys. 54, 2970-2982

12. Chang, J., Boley, C., Martinex, M. Molander, W., and Wamer, B. (1994) Beam characeristios of a large-bore copper laser with a radiatively cooled plasma, SPIE 2118, 2-8.

13. Chang, J. (1993) Pressure dependenoe of copper laser output characteristics, Applied Optics 32, 5230-5235.

14. Iseki, Y. Watanabe, I., and Noda, E. (1995) Output characteristics and improvement in beam-profile of largebore copper vapor tasers. SPIE

15. von Engle, A. (1965) lonized gases, Clarendon Press, Oxford.

16. Jaoob, J. and Mani, S., thermal instability in high-power laser discharge. Appl. Phys. Let. ?. 53-?.

17. Chang, J., Alger, T, Anderson, A., and Amold, A. (1994) New hollow-cathade elearode for high-power copper lasers, Digest of Conference on Lasers and Electro-Optics, 402

18. The majonity of data are from Optovac's Crystals Handbook. Absorplion data are from Hass. M. and Davisson, J. (1977) Visible and UV absorption in laser windows, Proceoding of The High Power Laser Optical Components and Component Material Meeting, Atlington, Visginia, 152.

19. Glover, A. Couts, D. Ramsay, D., and Piper, J. (1995) Progress in high-spoed UV micro-machining and highrepetition rate frequency-doubled copper vapor lasers, Proceeding ICALEO'94. 343-35I.

20. Molander. W. (1994) Improved efficiency of second-hammonic generation in BBO using the $511 \mathrm{~nm}$ line of an injoction-soeded copper laser. Digest of Conference on Lasers and Electro-Optics, 363.

"This work was performed under the auspices of the U.S. Department of Energy by Lawrence Livermore National Laboratory under contract No. W-7405-Eng-48. 